Ethik Med 2005 - 17:5-6 DOI 10.1007/s00481-005-0359-9 Online publiziert: 5. Februar 2005 c) Springer Medizin Verlag 2005
Gisela Bockenheimer-Lucius ${ }^{1} \cdot$ Matthias Kettner ${ }^{2}$

${ }^{1}$ Frankfurt a. M. ${ }^{2}$ Witten-Herdecke

\title{
Auf dem Weg zu einer „Gefälligkeitsmedizin“?!
}

as neue Jahr beginnt für unsere Zeitschrift mit einer Erweiterung der Schriftleitung. Die vielfältigen und für Patienten und Ärzte ebenso wie für die gesellschaftliche Entwicklung wichtigen Fragestellungen und Herausforderungen durch die Medizinethik werden innerhalb der Akademie für Ethik in der Medizin (AEM) inzwischen in einer Vielzahl von nationalen wie internationalen Projekten, Tagungen und Arbeitsgruppen bearbeitet und diskutiert. Über diese Arbeit der AEM wollen wir unsere Leser mit verstärkten Kräften informieren und freuen uns, Frau Marianne Rabe (Berlin), Frau Prof. Dr. Bettina Schöne-Seifert (Münster), Herrn Prof. Dr. Gerd Richter (Marburg) und Herrn Prof. Dr. Klaus Steigleder (Bochum) begrüßen zu dürfen.

Die gemeinsame Arbeit kommt natürlich in besonderer Weise in der Jahrestagung der AEM zur Geltung und wird diesmal das hochaktuelle Thema einer „wunscherfüllenden Medizin“ aufgreifen.

Die ärztlichen Aufgaben und Handlungsziele der Erhaltung oder Wiederherstellung der Gesundheit sowie der Linderung krankheitsbedingten Leidens sind verbunden mit der Notwendigkeit einer durch den Arzt gestellten medizinischen Indikation und dadurch mit Behandlungsmöglichkeiten, die vom Expertenurteil gedeckt sind. Dieses Verständnis rechtfertigt einerseits die Ausgrenzung all dessen, was gesetzlich Versicherte nicht beanspruchen können, weil es wegen mangelnder medizinischer Notwendigkeit in der Regel von den Kassen nicht bewilligt und von Ärzten nicht bewirkt werden soll. Andererseits rechtfertigt es aber auch die Verweigerung ärztlichen Handelns aufgrund fehlender medizinischer Begründung. Jedes therapeutische Handeln bedarf neben der Einwilligung des Patienten einer Indikation. Der Arzt muss dem Wunsch eines Patienten dann nicht nachgeben, wenn eine Behandlung schädlich ist, wenn sie unschädlich, aber sinnlos ist, wenn sie unverhältnismäßig aufwändig ist oder die persönliche Integrität des Arztes verletzt. Allerdings ist zu bedenken, dass bereits der Entscheidung über Sinnhaftigkeit, Zielvorstellung des medizinischen Handelns und Wahl der Mittel moralische Urteile zugrunde liegen.

Drei Entwicklungen erschüttern vor allem das vertraute Grundverständnis. Intensiver denn je wird zwar in den letzten Jahren die „Medikalisierung" des Lebens beklagt und besorgt der Trend zu einer „Gefälligkeitsmedizin“ konstatiert, dennoch konkurriert zum einen die herkömmliche Vorstellung von Medizin als Versorgung des kranken Menschen inzwischen deutlich mit der Nachfrage nach Optimierung des Lebensgefühls und der Lebensplanung. Das Ziel vieler „Kunden“ der modernen Fortpflanzungsmedizin sind z. B. Kinder, deren Anzahl, Entstehungszeitpunkt und Geschlecht besser zur eigenen Lebensplanung passen - nicht zu reden von Leihmutterschaft und anderen ethisch umstrittenen, gleichwohl von vielen Eltern gewünschten Fortpflanzungsmethoden.

Die unerhörte Nachfrage nach Schönheitschirurgie, Antiaging- und Lifestylemedizin entzweit die Ärzteschaft in Verächter und Verfechter. Einerseits ist die Zahl der in Krankenhäusern, Belegkliniken und Praxen durchgeführten ästhetisch-plastischen Operationen zwischen 1990 und 2002 auf rund 660.00o Eingriffe um das Sechsfache gestiegen. Andererseits hat der Präsident der Bundesärztekammer Jörg Hoppe zu einer Ärztekoalition gegen den Schönheitswahn aufgerufen. Macht sich die moderne Medizin zur Magd der Kulturindustrie? Ist eine Kampagne gegen „Schönheitswahn“ berechtigt?

Die zweite Entwicklung, die in das medizinische Selbstverständnis folgenreich eingreift, ist das Begehren nach medizinischen Utopien. Seit der Entschlüsselung des Genoms rückt die Sehnsucht nach Ver- 
besserung der gebrechlichen menschlichen Konstitution durch Geweberegenerierung, Stammzelltherapie, Keimbahnveränderung und Enhancement wieder in das Blickfeld.

Zum dritten wächst die Nachfrage der Patienten nach alternativen Heilmitteln, Diagnose- und Behandlungsverfahren, deren Wirksamkeit sich eher subjektiverweist. Akupunktur, natur-und pflanzenheilkundliche Verfahren, Homöopathie, traditionelle chinesische Medizin (TCM) und Anthroposophie befriedigen verbreitete Bedürfnisse nach einer ganzheitlichen, selbstbestimmten Auffassung von Gesundheit und Krankheit. Hier ist nicht nur eine interessante Grauzone entstanden, sondern ein auch für Heilpraktiker und andere Heilhilfspersonen großer und lukrativer Schattenmarkt.

Das Entgegenkommen der Ärzte, neuerdings auch der Kassen, ist beträchtlich und beginnt den Begriff der Schulmedizin zu verändern. Schon jeder zehnte von Deutschlands Ärzten führt Zusatzbezeichnungen im Umfeld der alternativen Methoden. Die Offenheit für das Alternative bis hin zur Quacksalberei, die Kundenorientierung und die hochgestimmte Heilungsvision sind Kategorien, denen sich die neue wunscherfüllende Medizin mehr und mehr öffnet.

Die Auseinandersetzung hat erst begonnen und bringt eine Fülle von Fragen mit sich. Die Jahrestagung der AEM wird sich der Problematik umfassend widmen. Unser "call for abstracts" in diesem Heft (der zeitliche Rahmen wurde mit Blick auf das Erscheinungsdatum der Zeitschrift bis zum 31. März 2005 erweitert) bietet zahlreiche Anregungen, sich der hochkomplexen Thematik zu widmen. Die AEM lädt herzlich zur Teilnahme ein.

\section{Korrespondierender Autor}

\section{Dr. med. Gisela Bockenheimer-Lucius}

Senckenbergisches Institut für Geschichte der Medizin, Theodor-Stern-Kai 7, 60590 Frankfurt am Main E-Mail: Bockenheimer-Lucius@em.uni-frankfurt.de 